\title{
DESARROLLO DE UN AMBIENTE DE APRENDIZAJE MEDIADO CON TIC PARA LA ENSEÑANZA DE LA EDUCACIÓN ECONÓMICA FINANCIERA
}

\section{DEVELOPMENT OF A LEARNING ENVIRONMENT USING ICTS FOR THE TEACHING OF ECONOMIC FINANCIAL EDUCATION}

\author{
MSc.(C)Diana Janneth Berdugo Portilla*, PhD. Julio Enrique Duarte** \\ PhD. Flavio Humberto Fernández Morales,*** \\ *Universidad Pedagógica y Tecnológica de Colombia, \\ E-mail: julioenriqued1@gmail.com. \\ ** Universidad Pedagógica y Tecnológica de Colombia,. \\ E-mail: flaviofm1@gmail.com.
}

Resumen: El objetivo del estudio consistió en la evaluación de conceptos básicos sobre matemáticas financieras, antes y después de cursar la materia con el uso de las TIC y sin ellas. La metodología del estudio fue cuantitativa, para lo cual se tuvo en cuenta un grupo control y otro experimental; la variable cuantitativa es el rendimiento académico. La población de estudio correspondió a estudiantes de grado sexto, donde se evaluaron dos grupos, cada uno de 25 estudiantes cuyas edades oscilan entre 10 y 11 años. Se realizó una prueba inicial que sirvió para proponer un ambiente de aprendizaje mediado por TIC. Los resultados indican que los estudiantes del grupo experimental mejoraron su desempeño pasando del $56 \%$ que aprobó inicialmente al $80 \%$ que lo hizo en la prueba final. Se puede concluir que un ambiente de aprendizaje mediado por TIC, complementado con una metodología que convine diversos escenarios educativos, y junto a estrategias didácticas motivadoras, los estudiantes obtendrán mejores resultados en sus evaluaciones.

Palabras clave: matemática financiera, ambientes virtuales de aprendizaje, TIC, educación económica y financiera..

\begin{abstract}
The objective of the study was to evaluate basic concepts in financial mathematics, before and after studying the subject with the use of ICT and without them. The methodology of the study was quantitative, for which a control group and an experimental group were considered; the quantitative variable is the academic performance. The study population corresponded to sixth grade students, where two groups were evaluated, each of 25 students aged 10 to 11 years. An initial test was used to propose an ICT-mediated learning environment. The results indicate that students in the experimental group improved their performance from $56 \%$ who initially approved to $80 \%$ who did so in the final test. It is possible to conclude that a learning environment, mediated by ICT, complemented by a methodology that agrees diverse educational scenarios, and together with motivational didactic strategies, students will obtain better results in their evaluations..
\end{abstract}

Keywords: Financial mathematics, virtual learning environments, ICT, economic education. 


\section{INTRODUCCIÓN}

El Ministerio de Educación Nacional colombiano, MEN, desde el año 2012 emprendió la implementación de un Programa de Educación Económica y Financiera, PEEF, en convenio con la Asociación Bancaria y de Entidades Financieras de Colombia, Asobancaria. El programa busca "Fomentar el pensamiento crítico en los niños, niñas, adolescentes y jóvenes mediante el desarrollo de saberes, habilidades, actitudes y valores que les ayude a reconocer, comprender, analizar y decidir responsablemente frente a fenómenos económicos y financieros presentes en su cotidianidad". (MEN, 2013)

La educación financiera ha cobrado especial importancia en una época de incertidumbre como en la que vivimos, donde la crisis económica y financiera iniciada en el año 2007 ha impactado en los niveles de empleo y por ello los individuos se encuentran en la obligación de tomar previsiones ante situaciones adversas (Xander et al., 2016; Hernández et al., 2014; Bustamante et al., 2013).

Además, la corrupción política y económica percibida por los niños y la filosofía del dinero rápido, generan una falta de ética en el planteamiento del esfuerzo, el trabajo y el ahorro, que pueden provocar situaciones aún más difíciles cuando estos niños alcancen la edad adulta (Del Brío et al., 2015; Núñez, 2015; Zuluaga, 2017).

Se espera que uno de los aportes de la EEF en nuestro país sea el fortalecimiento del bienestar de la sociedad, puesto que “...consumidores más educados y mejor informados toman mejores decisiones financieras a lo largo de su vida, las cuales no sólo favorecen sus intereses particulares sino que, en conjunto, ayudan a la estabilidad del sistema financiero al reducir la probabilidad de incidentes de crisis". (MEN, 2014)

La EEF en las instituciones educativas de nivel primaria, secundaria y media se ha ido incorporando lentamente de manera superficial, manifestándose una carencia de material pedagógico debido a los pocos años en que se ha implementado dicha temática (Villada et al., 2017; Moreno et al., 2017).

En la actualidad, los modelos educativos innovadores deben fomentar ambientes de aprendizaje interactivos, sincrónicos y asincrónicos, donde el docente se encuentre comprometido con el aprendizaje de sus alumnos y cumpla un papel como asesor y facilitador (García et al., 2017; Páez et al., 2016). En estos modelos, los estudiantes se convierten en actores de cambio con habilidades y modos de trabajo innovadores en los cuales utilizan tecnologías de vanguardia, materiales didácticos, recursos de información y contenidos digitales (Bernal, 2017; Piratoba y Rojas, 2014; Guzmán et al., 2014). Una propuesta metodológica para operar estos modelos educativos es la de Ambientes de Aprendizaje Mediados por TIC, ya que desarrollar un ambiente de este tipo no es trasladar la docencia de las aulas de clase a un ambiente virtual, sino mediarlas con las Tecnologías de la Información y la Comunicación, TIC (Tangarife, 2013; Riscanevo, 2016; Salcedo et al., 2017).

El Colegio Cooperativo Reyes Patria, de Sogamoso, Boyacá, posee una infraestructura propia para desarrollar este tipo de ambientes, ya que cuenta con salas especializadas y su propia plataforma. Además, el colegio tiene la necesidad de implementar el requerimiento del MEN sobre EEF, siendo necesario el desarrollo de un ambiente de aprendizaje para esta área.

El objetivo del estudio consistió en la evaluación de conceptos básicos sobre matemáticas financieras, antes y después de cursar la materia con el uso de las TIC y sin ellas. En este trabajo se observó la población objetivo, estudiantes del grado sexto de educación básica, valorando los conocimientos adquiridos, utilizando la plataforma de la institución y evaluando, a través de pruebas internas, los conceptos adquiridos en el área de matemática financiera.

\section{MARCO TEÓRICO Y METODOLOGÍA}

\subsection{Ambientes de aprendizaje}

El colegio donde se desarrolló la investigación cuenta con un modelo de aprendizaje basado en el constructivismo, el cual sirvió como base para la implementación del ambiente de aprendizaje propuesto. La idea central del constructivismo reside en que la elaboración del conocimiento constituye una modelización más que una descripción de la realidad. En otras palabras, puede 
denominarse teoría constructivista a aquella que entiende que el conocimiento es el resultado de un proceso de construcción o reconstrucción de la realidad, que tiene su origen en la interacción entre las personas y el mundo (Sepúlveda, 2015; Cárdenas y Martínez, 2015).

Según Chaupart et al. (2014), se puede definir un Ambiente de Aprendizaje como el conjunto de entornos de interacción, sincrónica y asincrónica, donde, con base en un programa curricular, se lleva a cabo el proceso enseñanza-aprendizaje a través de un sistema de administración del mismo. Adicionalmente, Angarita citada por Hernández et al., (2014), señalan que "un ambiente de aprendizaje constituye un espacio propicio para que los estudiantes obtengan recursos informativos y medios didácticos para interactuar y realizar actividades encaminadas a metas y propósitos educativos previamente establecidos".

\subsection{Educación Económica Financiera}

La Organización para la Cooperación y el Desarrollo Económico (OCDE, 2011) asume la educación financiera como "un proceso por medio del cual los individuos desarrollan los valores, los conocimientos, las competencias y los comportamientos necesarios para la toma de decisiones financieras responsables que requieren la aplicación de conceptos financieros básicos y el entendimiento de los efectos que los cambios en los principales indicadores macroeconómicos generan en su propio nivel de bienestar económico". Igualmente, (UNICEF, 2013) plantea que "La educación social y financiera tiene como objetivo inspirar a los niños a ser ciudadanos social y económicamente empoderados, dotándolos de las actitudes y los conocimientos necesarios para convertirse en agentes activos capaces de transformar sus comunidades y sociedades".

En la figura 1 se muestran los componentes que la UNICEF ha predeterminado para la educación financiera en niños y jóvenes, dependiendo del nivel de edad y la educación a implementar. Este proyecto se basará en el nivel 3, de edades entre 10 y 14 años, en el cual se destaca que la población de estudio deben ser consumidores informados y que sean coherentes frente a los riesgos financieros a los que se vean enfrentados.

\begin{tabular}{|c|c|c|c|}
\hline & $\begin{array}{c}\text { Educación social/ } \\
\text { habilidades para la vida }\end{array}$ & Educación financiera & $\begin{array}{l}\text { Educación sobre } \\
\text { los medios de vida } \\
\text { sostenibles }\end{array}$ \\
\hline $\begin{array}{l}\text { Nivel 1: } \\
0-5 \text { años }\end{array}$ & $\begin{array}{l}\text { Emociones, consecuencias, } \\
\text { salud/seguridad, compasión }\end{array}$ & $\begin{array}{l}\text { Valor de dinero, precios, } \\
\text { ahorro, posesiones }\end{array}$ & \multirow{2}{*}{$\begin{array}{l}\text { Intereses profesionales, } \\
\text { profesiones, espiritu } \\
\text { empresarial, metas, } \\
\text { iniciativa, capacidad } \\
\text { para la resolución de } \\
\text { problemas, trabajo en } \\
\text { equipo, recibir consejos, } \\
\text { evitar riesgos }\end{array}$} \\
\hline $\begin{array}{l}\text { Nivel 2: } \\
\text { 6-9 años }\end{array}$ & $\begin{array}{l}\text { Los derechos del niño, las } \\
\text { responsabilidades, el respeto } \\
\text { por los demás, las reglas, las } \\
\text { capacidades auditivas }\end{array}$ & $\begin{array}{l}\text { Necesidades y deseos, plan } \\
\text { de ahorro, recompensas, } \\
\text { reconocer los bancos y los } \\
\text { servicios financieros }\end{array}$ & \\
\hline $\begin{array}{l}\text { Nivel 3: } \\
\text { 10-14 } \\
\text { años }\end{array}$ & $\begin{array}{l}\text { Expresar opiniones, trabajo } \\
\text { en equipo, capacidades de } \\
\text { investigación, el aprecio por } \\
\text { un aprendizaje permanente }\end{array}$ & $\begin{array}{l}\text { Consumidor informado, } \\
\text { planificación a corto plazo } \\
\text { frente a largo plazo, riesgos } \\
\text { financieros, fectos de la } \\
\text { publicidad }\end{array}$ & $\begin{array}{l}\text { Vocaciones, } \\
\text { oportunidades, plan de } \\
\text { acción, autodisciplina, } \\
\text { perseverancia, } \\
\text { comunicación }\end{array}$ \\
\hline $\begin{array}{l}\text { Nivel 4: } \\
15+\text { años }\end{array}$ & $\begin{array}{l}\text { Injusticia social, gestión del } \\
\text { tiempo, relaciones, liderazgo }\end{array}$ & $\begin{array}{l}\text { Capacidades de } \\
\text { negociación, poder } \\
\text { adquisitivo, tipos de } \\
\text { interés, delitos financieros }\end{array}$ & $\begin{array}{l}\text { Salarios, necesidades } \\
\text { de capital, mercadeo, } \\
\text { empleabilidad, adaptación } \\
\text { al cambio, capacidad de } \\
\text { gestión }\end{array}$ \\
\hline
\end{tabular}

Fig. 1. Marco internacional de la educación financiera para los niños y jóvenes (UNICEF, 2013).

En general, como lo plantean Gnan et al. (2007), las definiciones de EEF encontradas en la mayoría de programas y estrategias alrededor del mundo, tienen tres elementos en común: pretenden generar mayor comprensión y capacidad para la toma de decisiones; propician el reconocimiento de la interrelación de las personas con el sistema financiero $y$, finalmente, incorporan características y condiciones del contexto social en el que las personas se empoderan para tomar decisiones financieras, que les permitan transformar sus contextos desde una perspectiva social y económica.

\subsection{Matemática Financiera.}

Según Mehl (1964), la EEF permite modelar la realidad y emplear un sentido lógico para arribar a las generalizaciones a través de la simbolización. En consecuencia, la asignatura Matemática Financiera está orientada a estimular el desarrollo de destrezas y habilidades cognoscitivas, que en fase posterior se traduce en capacidades analíticas y críticas.

Desde el punto de vista matemático, la base de la Matemática Financiera radica en explorar el cambio que se genera en uno o varios capitales a través del tiempo.

La importancia de la Matemática Financiera radica en la teoría del valor, trabajo desarrollado por (Ricardo, 1959), quien explica que los precios eran consecuencia de la cantidad de trabajo que se necesitaba para producir un bien. Marx (1975) adopta esta teoría y otras dos corrientes: la 
dialéctica hegeliana y la exposición de la revolución industrial, para realizar una genial síntesis de la teoría del valor, es decir, la transformación de la mercancía en dinero.

\subsection{Diseño Metodológico}

El diseño metodológico que se empleó es un diseño experimental de observación cuantitativa, para lo cual se tendrá un grupo control y otro experimental; la variable cuantitativa es el rendimiento académico calculado por pruebas internas de la institución.

El proyecto se adelantó en el Colegio Cooperativo Reyes Patria, de Sogamoso, Boyacá. Es un establecimiento educativo de carácter privado, mixto y de orientación católica, que ofrece todos los niveles educativos, desde preescolar hasta la media. El colegio cuenta con aproximadamente 970 estudiantes e instalaciones apropiadas, donde cada salón cuenta con video beam, tv y la plataforma Aula Virtual.

La población de estudio se centró en estudiantes de grado sexto, donde se evaluaron dos grupos, cada uno de 25 estudiantes, de estrato 2 a 4 del municipio de Sogamoso y su edad oscila entre $10 \mathrm{y}$ 11 años; están conformados por 14 niñas y 11 niños, respectivamente.

El objetivo del análisis fue el de evaluar conceptos básicos sobre matemática financiera, antes $\mathrm{y}$ después de cursar la materia con el uso de las TIC y sin ellas. En este trabajo se observó la población objetivo valorando los conocimientos adquiridos, utilizando la plataforma de la institución y evaluando, a través de pruebas internas, los conceptos adquiridos en el área de matemática financiera.

La investigación se desarrolló en cuatro fases, a saber:

i. Identificación de las temáticas EEF, y demás requerimientos pedagógicos y técnicos, necesarios para la creación de un Ambiente de Aprendizaje Mediado por TIC, orientado a los estudiantes del grado sexto del Colegio Cooperativo Reyes Patria de la ciudad de Sogamoso.

ii. Diseño de un Ambiente de Aprendizaje Mediado por TIC para la enseñanza de la EEF en estudiantes de grado sexto, que haga uso de los recursos tecnológicos existentes en la Institución. iii. Aplicación y valoración del Ambiente de Aprendizaje Mediado por TIC, con la población objeto de estudio, para evaluar su eficacia en el Aprendizaje EEF y proponer mejoras.

iv. Análisis y evaluación de resultados obtenidos en la investigación.

El ambiente de aprendizaje se implementó en la asignatura de Estadística, como una competencia a desarrollar dentro de ella

\section{Resultados y discusión}

\subsection{Diseño del ambiente de aprendizaje}

Se aplicó una prueba diagnóstica que constó de 12 preguntas de selección múltiple, donde se evaluaron los conocimientos previos acerca de esta materia (conceptos básicos en primaria) y se aplicó a 50 estudiantes, los cuales se encontraban divididos en dos grupos. El primer grupo arrojó que el $84 \%$ de los estudiantes poseen conceptos básicos elementales de esta materia; mientras en el otro grupo, tan solo el $56 \%$ aprobaron la prueba, como se muestra en la figura 2; por lo anterior se decidió que el grupo experimental fuera el que mayor carencia tenía de estos conceptos.

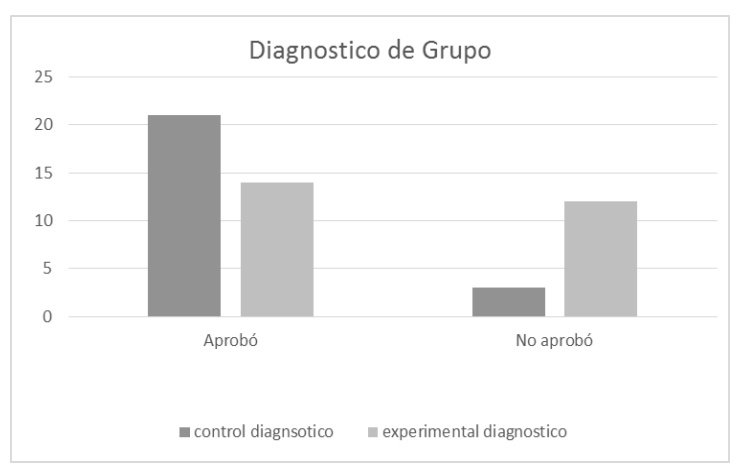

Fig. 2. Resultado evaluación conceptos Básicos.

Realizado el análisis de la prueba diagnóstica, donde se identificó la falta de conocimientos de algunos estudiantes, se procedió a establecer los componentes básicos que intervienen en la experiencia de aula. En este caso los actores que intervienen en el ambiente son: los estudiantes de grado sexto y profesores, mientras que la 
mediación se efectúa a través de la plataforma de la institución

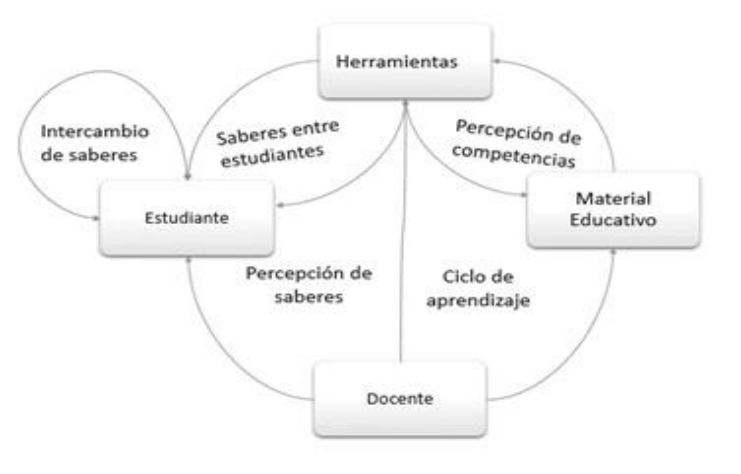

Fig.3.Componentes pedagógicos de la transposición didáctica

Se tomaron como herramientas el chat, foros, desarrollo de ejercicios, visualización de videos e interacción con otras páginas web relacionadas con el tema y material destinado al trabajo independiente, ver figura 3. Para el componente pedagógico se emplean los lineamientos del MEN.

Las herramientas virtuales y los lineamientos curriculares aquí utilizados, son de uso frecuente en la enseñanza mediada por ambientes virtuales (Barrera et al., 2017; Niño et al., 2016).

El objetivo principal del ambiente de aprendizaje es contribuir al desarrollo de las competencias básicas de las matemáticas financieras, el cual se estructuró como se muestra en la figura 4, implementado en la plataforma del Colegio Reyes Patria, de la ciudad de Sogamoso.

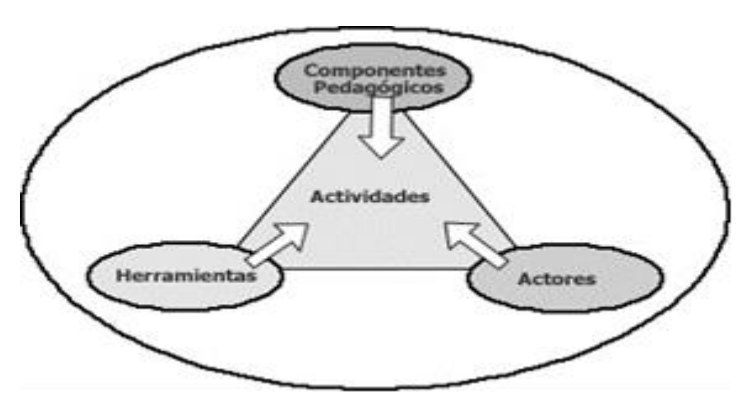

Fig. 4. Ambiente de aprendizaje diseñado

En la figura 4 se observa la interacción con cada uno de los actores en el ambiente de aprendizaje, donde las herramientas utilizadas están basadas en foros, enlaces, chat y los ejercicios en línea que complementan los saberes entre estudiantes, material creado para desarrollar cada una de las competencias establecidas; el docente puede establecer el nivel del conocimiento adquirido en ella a través del desarrollo de las guías de aprendizaje y la evaluación final.

El intercambio de saberes entre estudiantes se establece en el momento de desarrollar las guías de aprendizaje, trabajadas en clase con la asesoría del docente, y el resultado de los foros desarrollados en la plataforma. El material educativo se estructuró a partir de las competencias básicas exigidas por el MEN y apoyados en el material que algunas entidades financieras han aportado para este fin.

El objetivo es contribuir a la adquisición de las competencias asignadas por el MEN para la asignatura de matemáticas financieras en estudiantes de grado sexto, donde se resaltan los siguientes desempeños conceptuales, que se constituyen en el eje principal de la temática:

- Relaciona el consumo responsable de bienes y servicios con los hábitos financieros para mejorar la calidad de vida.

- Explica cómo, en las finanzas familiares, el presupuesto facilita la adecuada administración del consumo responsable de bienes y servicios

- Evalúa la importancia del ahorro, la inversión y el consumo responsable de bienes y servicios para el cumplimiento de metas en el mejoramiento de su entorno familiar.

- Describe si se justifica endeudarse para adquirir bienes y servicios según los ingresos familiares.

- Establece estrategias para cumplir metas a través del desarrollo de hábitos financieros responsables que influyen en el bienestar propio y de los demás.

\subsection{Actividades desarrolladas}

El trabajo desarrollado se fundamenta en varias actividades dentro de la plataforma institucional como se muestra en la figura 5. Estas actividades constan de foros, ejercicios en línea, tareas, documentos, evaluaciones y enlaces que complementan la teoría en el aula de clase. 


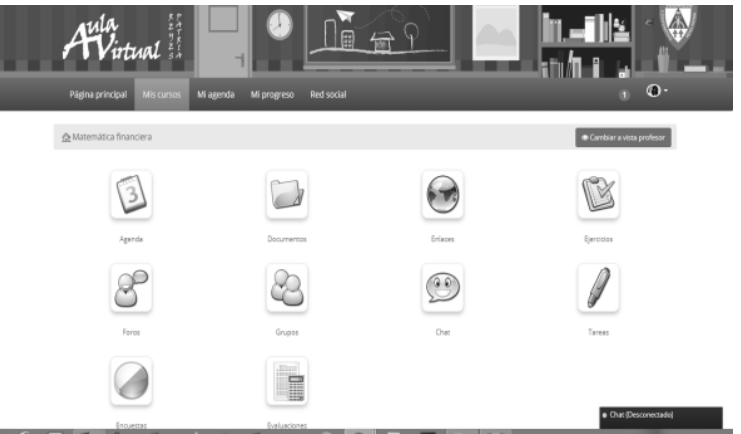

Fig. 5. Captura de pantalla de las actividades en el aula virtual

El trabajo con los estudiantes se desarrolló en varios ambientes como: salón de clase, biblioteca y sala de informática. Las actividades desarrolladas en el aula se realizaron en parejas (grupo experimental), donde algunos estudiantes, para el desarrollo de sus actividades, investigaron en la red y otros en sus hojas guías de trabajo.

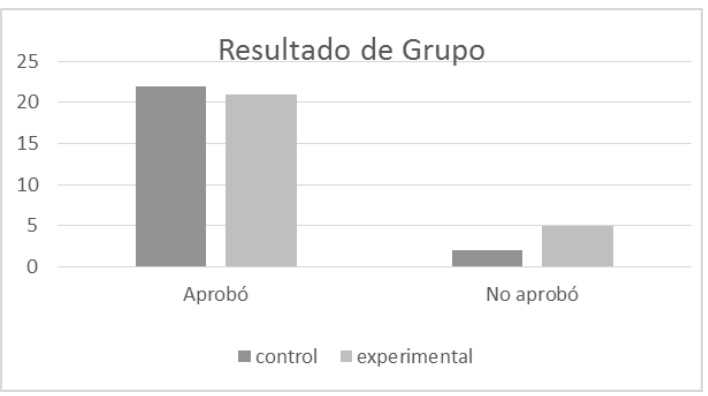

Fig. 6. Resultado final.

Al finalizar la aplicación del ambiente de aprendizaje se realizó la prueba final escrita, como se ve en la figura 6. Comparando este resultado con lo obtenido en la prueba diagnóstica, figura 2 , se puede verificar que los estudiantes mejoraron su desempeño, pasando del $56 \%$ que aprobó inicialmente al $80 \%$ que lo hizo en la prueba final.

El grupo control, luego de aplicar la metodología tradicional, no sufrió cambios significativos en cuanto a la cantidad de estudiantes que pasaron la prueba. Al comparar el rendimiento de los grupos experimental y control, en la prueba final, se observa que el uso de las TIC aporta significativamente en la mejora de los conocimientos de los estudiantes en EEF, lo cual muestra que el ambiente de aprendizaje y la metodología utilizada fueron adecuadas para fortalecer estos conocimientos

\section{Conclusiones}

La implementación de un ambiente de aprendizaje, mediado con TIC, debe motivar al estudiante, y al docente, para de salir de su rutina diaria de tablero y marcador.

En este caso los resultados muestran que un ambiente de aprendizaje, mediado por TIC, complementado con una metodología que convine diversos escenarios educativos, y junto a estrategias didácticas motivadoras, los estudiantes obtendrán mejores resultados en sus evaluaciones.

El ambiente de aprendizaje debe acoplarse a las condiciones naturales o propias del entorno, de modo que se apoye al estudiante en alcanzar las metas de aprendizaje que se hayan definido. En este sentido, el docente debe tener las competencias en el uso de las TIC que le permitan adaptarse a las herramientas disponibles en la institución para optimizar su integración en el proceso de enseñanza aprendizaje.

El ambiente para el aprendizaje de la EEF, aquí propuesto, se puede replicar en otras instituciones, con poblaciones estudiantiles diferentes, con el fin de convalidar su eficacia en el aula.

\section{REFERENCIAS}

Barrera M, C. E., Fernández M, F. H., \& Duarte, J. E. (2017). Diseño de un ambiente de aprendizaje mediado por TIC para la enseñanza de operadores mecánicos orientado al grado séptimo de la educación básica, en el Colegio Boyacá de Duitama. Revista Colombiana de Tecnologías de Avanzada, 2 (30). doi: https://doi.org/10.24054/16927257.v30.n3 0.2017 .2740

Bernal , M. L. (2017) ¿qué escriben los niños?, una mirada desde el modelo escuela nueva. Revista de investigación, Desarrollo e innovación, 7 (2), 255-268. doi:

https://doi.org/10.19053/20278306.v7.n2. 2017.6069

Bustamante Z, L. F., Porto P, I. A., y Hernández T, F. (2013). Gestión estratégica de las áreas funcionales de la empresa: una perspectiva competitiva internacional. Revista de Investigación, Desarrollo e Innovación, 4 (1), 56-68. doi: 10.19053/20278306.2607 
Cárdenas S, R. N., y Martínez C, D. (2015). El paisaje sonoro, una aproximación teórica desde la semiótica. REVISTA DE INVESTIGACIÓN, DESARROLLO E INNOVACIÓN， 5(2), 129-140. doi: https://doi.org/10.19053/20278306.3717

Chaupart, J. M., Corredor, M. V., y Marín, G. (2014). El tutor, el estudiante y su nuevo rol. Guadalajara: Desarrollo de ambientes de aprendizaje en educación a distancia. Universidad de Guadalajara. Coordinación de Educación Continua, Abi. Recuperado de:

http://investigacion.ilce.edu.mx/panel_con trol/doc/Rayon_Parra.pdf

Del Rrío, E., López, C., y Vereas, C. (2015). Educación financiera en la infancia. Propuesta didáctica en Educación Infantil. ENSAYOS, Revista de la Facultad de Educación de Albacete, 30(2). Recuperado de: http://www.revista.uclm.es/index.php/ensa yos

García Q, B., Coronado, A., y Giraldo O, A. (2017). Implementación de un modelo teórico a Priori de competencia matemática asociado al aprendizaje de un objeto matemático. REVISTA DE INVESTIGACIÓN, DESARROLLO E INNOVACIÓN, 7(2), 301-315. doi:https://doi.org/10.19053/20278306.v7. n2.2017.6072

Gnan, E., Silgoner, M., \& Weber, P. (2007). Economic and Financial Education:Concepts, Goals and Measurement. Austrian Central Bank Monetary.

Guzmán L, J., Torres, I., y Alvarez, J. F. (2014). Propuesta de un generador de aplicaciones educativas basadas en televisión digital usando arquitectura de cómputo en la nube. Revista Colombiana de Tecnologías de Avanzada, 2 (24).

Hernández T, F. D., Bustamante Z, L. F., y Porto P, I. A. (2014). Evaluación del grado de competitividad de cinco empresas del sector lechero de Sincelejo, Colombia. Revista de Investigación, Desarrollo e Innovación, 5 (1), 8-19. https://doi.org/10.19053/20278306.3135

Hernández S, C., Prada N, R., y Gamboa S, A. (2017). Conocimiento y uso del lenguaje matemático en la formación inicial de docentes en matemáticas. REVISTA DE INVESTIGACIÓN, DESARROLLO E INNOVACIÓN, 7(2), 287-299. doi:https://doi.org/10.19053/20278306.v7. n2.2017.6071

Marx, K. (1975). El capital. Madrid, España: Siglo XXI.

Mehl, L. . (1964). Elementos de Ciencia Fiscal. Barcelona, España: Bosch

Ministerio de Educación Nacional, MEN. (2013). orientaciones pedagógicas para la educación económica y financiera. Recuperado de: http://www.mineducacion.gov.co/1621/art icles-

340033_archivo_pdf_Orientaciones_Edu_ economica_financiera.pdf

Ministerio de Educación Nacional, MEN. (2014). Guías Pedagógicas para la convivencia escolar. Bogotá.

Moreno G, E., García S, A., y Gutiérrez D, L. (2017). Nivel de educación financiera en escenarios de educación superior. Un estudio empírico con estudiantes del área económico-administrativa. Revista Iberoamericana de Educación Superior, $8(22)$.

doi: http://dx.doi.org/10.22201/iisue.20072872 e.2017.22.234

Niño V, J. A., Martínez D, L. Y., y Fernández M, F. H. (2016). Mano robótica como alternativa para la enseñanza de conceptos de programación en Arduino. Revista Colombiana de Tecnologías de Avanzada, 2 (28), 132-139.

Núñez P, V. (2015). Pedagogía social e interculturalismo: una lectura posible. Revista de Investigación, Desarrollo e Innovación, 5 (2), 141-149. doi: $10.19053 / 20278306.3716$

OECD (2011). Guidelines on financial education at school and guidance.

Páez B, E., Corredor C, E., y Fonseca C, J. (2016). Evaluación del uso de herramientas sincrónicas y asincrónicas en procesos de formación de las ciencias agropecuarias. Revista Ciencia y Agricultura, 13(1), 7790. doi:https://doi.org/10.19053/01228420.48 08

Piratoba G, R. P., y Rojas M, C. E. (2014). Cambios en las concepciones iniciales e inducidas sobre la naturaleza de las matemáticas y su didáctica, en estudiantes de un programa de Licenciatura en Matemáticas y Estadística. Revista de Investigación, Desarrollo e Innovación, 5 
(1), 32-45. doi: https://doi.org/10.19053/20278306.3140

Ricardo, D. (1959). Principios de economía política y tributación. México, México: Fondo de Cultura Económica.

Riscanevo E, L. (2016). La teoría de la práctica social del aprendizaje en la formación de profesores de matemáticas. REVISTA DE INVESTIGACIÓN, DESARROLLO E INNOVACIÓN, 7(1), 93-110. doi:https://doi.org/10.19053/20278306.v7. n1.2016.5635

Salcedo R, R. Y., Fernández M, F. H., y Duarte, J. E. (2017). Unidad didáctica para la enseñanza de probabilidad mediada por un OVA, orientada a un colegio rural del municipio de Paipa. Revista Colombiana de Tecnologías de Avanzada, 2 (30). doi: https://doi.org/10.24054/16927257.v30.n3 0.2017 .2739

Sepúlveda D, O. (2015). Estudio del conocimiento didáctico - matemático del profesor universitario: un marco teórico de investigación. Revista de Investigación, Desarrollo e Innovación, 6 (1), 29-43. doi: http://doi.org/10.19053/20278306.4048

Tangarife Ch, D. (2013). Desarrollo de una aplicación web para el montaje de una mesa quirúrgica en el área de traumatología. REVISTA DE INVESTIGACIÓN, DESARROLLO E INNOVACIÓN, 4(1), 32-44. doi: https://doi.org/10.19053/20278306.2124

UNICEF (2013). Educación social y financiera para la infancia. Recuperado de: https://www.unicef.org/cfs/files/CFS_Fin Ed_Sp_Web_8_5_13.pdf

Villada, F., López L, J. M., y Muñoz G, N. (2017). El Papel de la Educación Financiera en la Formación de Profesionales de la Ingeniería. Formación universitaria, 10(2), 13-22. doi: https://dx.doi.org/10.4067/S071850062017000200003

Xander, P., Haydu, V. B., y de Souza, S. R. (2016). "DimDim: Negociando \& Brincando" no ensino de habilidades monetárias a préescolares. Revista CES Psicología, 9(1), 89-108.

Zuluaga D, J. F. (2017). Relación entre conocimientos, saberes y valores: un afán por legitimar los saberes más allá de las ciencias. Revista de Investigación, Desarrollo e Innovación, 8(1), 61-76. doi: 10.19053/20278306.v8.n1.2017.5973 